\title{
Whiting 1: Confirmation of its accretion by the Milky Way
}

\author{
Julio A. Carballo-Bello ${ }^{1,2, \star}$, Jesús M. Corral-Santana ${ }^{3,1}$, David Martínez-Delgado ${ }^{4}$, Antonio \\ Sollima ${ }^{5}$, Ricardo R. Muñoz ${ }^{6}$, Sonia Duffau ${ }^{2,1}$, and Márcio Catelan ${ }^{1,2}$ \\ ${ }^{1}$ Pontificia Universidad Católica de Chile, Instituto de Astrofísica, Av. Vicuña Mackenna 4860, 782-0436 \\ Macul, Santiago, Chile \\ ${ }^{2}$ Millennium Institute of Astrophysics, Santiago, Chile \\ ${ }^{3}$ European Southern Observatory, Alonso de Córdova 3107, Casilla 19001, Santiago, Chile \\ ${ }^{4}$ Astronomisches Rechen-Institut, Zentrum für Astronomie der Universität Heidelberg, Mönchhofstr. 12-14, \\ D-69120 Heidelberg, Germany \\ 5 INAF Osservatorio Astronomico di Bologna, via Ranzani 1, I-40127 Bologna, Italy \\ ${ }^{6}$ Departamento de Astronomía, Universidad de Chile, Camino El Observatorio 1515, Las Condes, Santiago, \\ Chile
}

\begin{abstract}
We investigate the association of Whiting 1 with the Sagittarius tidal stream by obtaining radial velocities for a sample of 101 stars observed with VIMOS. Our results reveal the presence of a component of the Sagittarius tidal stream with a radial velocity and distance - compatible with that of the globular cluster. Therefore, we conclude that Whiting1 was formed in the interior of the Sagittarius dwarf spheroidal galaxy and later accreted by the Milky Way. In addition, our data also reveal the detection for the first time of an ancient wrap of the Sagittarius tidal stream along the same line-of-sight and at the same heliocentric distance.
\end{abstract}

\section{Introduction}

The accretion of massive satellites is believed to have contributed to the formation of a significant fraction of galaxy haloes by transferring a large amount of gas, stars and globular clusters (GC). Compelling evidence for the latter comes from the GC systems of nearby galaxies (e.g. M31), where a large fraction of their globulars seems to align with tidal streams (e.g., [8]). As for the Milky Way, the accretion of GCs in the hierarchical formation scheme has been long proposed because of the existence of at least two distinct subgroups in the Galactic halo (e.g., [6]).

The most pronounced accretion event in the Galaxy is the one corresponding to the Sagittarius (Sgr; [3]) dwarf spheroidal and its associated stellar structure, the so-called Sgr tidal stream (e.g., [4]). Given the spatial extent of that system, it seems likely that some of the Galactic GCs might have formed within Sgr and been later accreted by the Milky Way.

\section{Observations and results}

In order to confirm the association of Whiting1 with the Sgr tidal stream, unveiled around the cluster by [1], we have observed 101 stars from our photometric catalogs with VLT+VIMOS. We have

^jcarballo@astro.puc.cl 


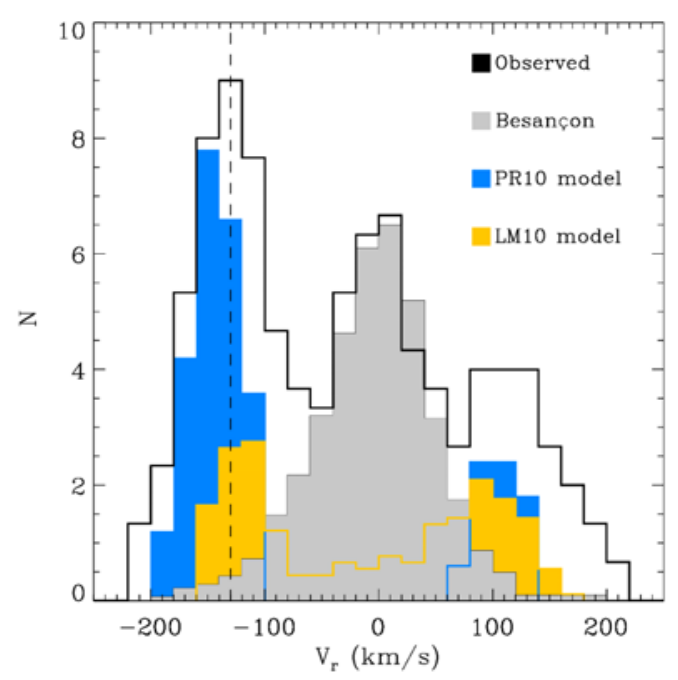

Figure 1. Figure taken from [2]. Radial velocity distribution obtained for the sample of stars around Whiting 1 (black solid line). The grey area indicates the velocity distribution as predicted by the Besançon model for the same line-of-sight, while the blue and yellow areas represent the predicted velocities by [7] and [5] models, respectively. The vertical line indicates the radial velocity of the GC

derived radial velocities for the targets by cross-correlating their spectra with a sample of templates corresponding to all spectral types and the obtained radial velocity distribution is shown in Figure 1. Three different components are clearly observed with approximate radial velocities $v_{r} \approx-130,0$ and $+150 \mathrm{~km} \mathrm{~s}^{-1}$. The expected Galactic kinematics for that region of the sky according to the synthetic Milky Way model of Besançon suggests that the central peak is generated by Galaxy stars. On the other hand, two additional components are found and the most prominent, located at negative velocities, is compatible with the radial velocity measured for the globular Whiting 1 . Therefore, our results confirm that Whiting1 is coincident in phase-space with the trailing arm of the Sagittarius tidal stream, being the first photometric+spectroscopic evidence of this association. The additional peak with positive radial velocities might correspond to the first detection of the predicted "old" leading arm of Sagittarius along that line-of-sight.

\section{References}

[1] Carballo-Bello, Sollima, A., Martínez-Delgado, D., et al., MNRAS, 445, 2971 (2014)

[2] Carballo-Bello, J.A., Corral-Santana, J. M., Martínez-Delgado, D., Sollima, A., Muñoz, R. R., Duffau, S., Catelan, M., \& Grebel, E. K. , MNRAS, 467, L91 (2017)

[3] Ibata, R. A., Gilmore, G., \& Irwin, M. J., Nature, 370, 194 (1994)

[4] Koposov, S. E., Belokurov, V., Evans, N. W., et al., ApJ, 750, 80 (2012)

[5] Law, D. R., \& Majewski, S. R., ApJ, 714, 229 (2010)

[6] Leaman, R., VandenBerg, D. A., \& Mendel J. T., MNRAS, 436, 122 (2013)

[7] Peñarrubia, J., Belokurov, V., Evans, N. W., et al., MNRAS, 408, 26 (2010)

[8] Veljanoski, J., Mackey, A. D., Ferguson, A. M. N., et al., MNRAS, 442, 2929 (2014) 